\title{
BUILDING A LEXICAL DOMAIN MAP FROM TEXT CORPORA
}

\author{
Tomek Strzalkowski
}

\author{
Courant Institute of Mathematical Sciences, New York University \\ 715 Broadway, rm. 704, New York, NY 10003, tomek@cs.nyu.edu
}

\begin{abstract}
SUMMARY
In information retrieval the task is to extract from the database all and only the documents which are relevant to a user query, even when the query and the documents use little common vocabulary. In this paper we discuss the problem of automatic generation of lexical relations between words and phrases from large text corpora and their application to automatic query expansion in information retrieval. Reported here are some preliminary results and observations from the experiments with a 85 million word Wall Street Journal database and a 45 million word San Jose Mercury News database (parts of 0.5 billion word TIPSTER/TREC database).
\end{abstract}

\section{INTRODUCTION}

The task of information retrieval is to extract relevant documents from large collection of documents in response to a user's query. When the documents contain primarily unrestricted text (e.g., newspaper articles, legal documents, etc.) the relevance of a document is established through 'full-text' retrieval. This has been usually accomplished by identifying key terms in the documents (the process known as 'indexing') which could then be Inatched against terms in queries (Salton, 1989). The effectiveness of any such lenn-based approach is directly related to the accuracy with which a set of terms represents the content of a document, as well as how well it contrasts a given document with respect to other documents. In other words, we are looking for a representation $R$ such that for any text items $D I$ and $D 2, R(D I)=R(D 2)$ iff meaning $(D I)=$ meaning $(D 2)$, at an appropriate level of abstraction (which may depend on types and chatainter of anticipated queries).

For all kinds of terms that can be assigned to the representation of a document, e.g., words, operatorargument pairs, fixed phrases, and proper names, various levels of "regularization" are needed to assure that syntactic or lexical variations of input do not obscure underlying semantic uniformity. Without actually doing semantic analysis, this kind of normalization can be achieved through the following processes: ${ }^{1}$

(1) morphological stemming: e.g., retrieving is reduced to retriev;

1 An altemative, but less efficient method is to generate all variants (lexical, syntactic, etc.) of words/plarases in the queries (SparckJones \& Tait, 1984).
(2) lexicon-based word nomalization: c.g., retrieval is reduced to retrieve;

(3) operator-argument representation of phrases: e.g., information retrieval, retrieving of information, and retrieve relevant information are all assigned the sume representation, retrieve +information;

(4) context-based term clustering into synonymy classes and subsumption hierarchies: e.g., fakeover is a kind of acquisition (in business), and Fortran is a programming language.

We have established the general architecture of a NLP-IR system that accommodates these considerations. In a general view of this design, depicted schematically below, an advanced NLP module is inserted between the textual input (new documents, user queries) and the database search engine (in our case, NIST's PRISE system).

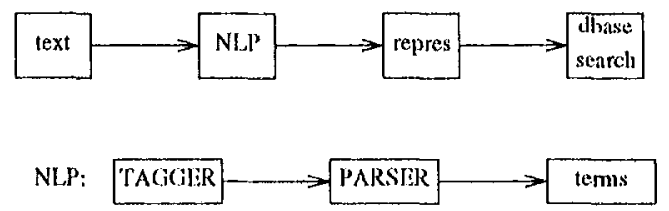

This design has already shown some promise in producing significantly better performance than the base statistical system (Strzalkowski, 1993). Its practical significance stems in no sinall part from the use of a fast and robust parser, TTP, which can process unrestricted text at speeds below $0.2 \mathrm{sec}$ per sentence. TTP's output is a regularized representation ol each sentence which reflects logical predicale-atgument struclure, e.g. logical subject and logical objects are identified depending upon the main verb subeategorization frame. For example, the verb abide has, among others, a subcategorization frame in which the object is a prepositional phratse with by, i.c.,

\section{ABIDE: subject NP object PREP by NP}

Subcategorization information is read from the on-line Oxford Advanced Learner's Dictionary (OALD) which TTP uses.

2 TTP stands for Tagged Text Parser, and it has been described in detail in (Stralkowski, 1992) and evaluated in (Stranlkowski \& Solleyen, 1993). 


\section{HEAD-MODIFIER STRUCTURES}

TTP parse structures are passed to the pharase extraction module where head+modifier (including predicate+argument) pairs are extracted and collected into occurrence patterns. The following types of head+modifier pairs are extracted:

(1) a head noun and its left adjective or noun adjunct,

(2) a head noun and the head of its right adjunct,

(3) the main verb of a clatuse and the head of its object phrase.

These types of pairs account for most of the syntactic variants for relating two words (or simple phrases) into pairs carrying compatible semantic content. For example, the pair retrieve tinformation will be extracted from any of the following fragments: information retrieval system; retrieval of information from databases; and information that can be retrieved by a user-controlled interactive search process. ${ }^{3}$

Figure 1 shows TTP parse and head+modifier pairs extracted. Whenever multiple-noun strings (two nouns plus another noun or adjective) are present, they need to be structurally disambiguated before any pairs can be extracted. This is accomplished using statistically-based preferences, e.g., world third is preferted to either country+world or country+third when extracled from third world country. If such preferences camnot be com. puted, all alternatives are discarded to avoid noisy input to clustering programs.

\section{TERM CORRELATIONS FROM TEX'T}

Head-modifier pairs serve as occurrence contexts for terms included in them: both single words (as shown in Figure 1) and other pairs (in case of nested pairs, e.g., cotumtry + /world +third /). If two terms tend to be modified with a number of common modifiers but otherwise appear in few distinct contexts, we assign them a similarity coefficient, a real number between 0 and 1 . The similarity is determined by comparing distribution characteristics for both terms within the corpus: in general we will credit high-content terms appearing in mulliple identicial contexts, provided that these contexts are not too commonplace. ${ }^{4}$ Figure 2 shows examples of terms sharing a number of common contexts along with frequencies of occurrence in a 250 MByte subset of Wall Street Journal database. A head context is when two distinct modifiers are attached to the same head element; a mod context is when the same term modifies two distinct heads.

To compute term similarities we used a variant of weighted Jaccard's measure described in e.g., (Grefen-

${ }^{3}$ subject+verb pairs are also extracted but these are not used in the lexical clustering procedure described here.

1 It would not be appropriate to predict similarity luetwees language and logarithm on the basis of their co-cecurrence with maturat.
[Sin) Jose Mercury News 08/30/91 [Business Section]

For McCaw, it would have hurt the company's strategy

of buidling a seamless mational cellular network.

[assert,

[Iwill_aux],Ifperl,[have],

[lverb, \{huti]),

[suljject, $\{\mathrm{np}, \mid \mathrm{n}, \mathrm{it}]\}]_{2}$

|objecl,|np),\{n,strategy),\{t_pos,the], [n_pos,|poss, |n,companyl|]. [of,

[|verb,[louild]],

|subject,inlyome|,

[object,|up,[n, netwotk\},[t_[pos,al],

|adj,|seamless||, [adj,Juational]], [adj,_cellular]\}]|!]|!]!],

[for, [n], [name, |mcenw]||]|].

\section{EXTRACTLI) PAIRS:}

$\begin{array}{ll}\text { hutt+si rategy } & \text { strategy+company } \\ \text { buildtnetwotk } & \text { network+cellulat } \\ \text { network+national } & \text { network+seamless }\end{array}$

Higure 1. Extracting Head+Moditier pairs from parsed sentences.

\begin{tabular}{|c|c|c|c|c|c|}
\hline \multirow[t]{2}{*}{ TERMI } & \multirow[t]{2}{*}{ JIRM2 } & \multicolumn{2}{|c|}{ COMM CNIXI' } & \multirow[t]{2}{*}{ HRQ! } & \multirow[t]{2}{*}{$\mathrm{RO} Q 2$} \\
\hline & & IIEAD & MOD & & \\
\hline \multirow[t]{5}{*}{ vice } & deputy & president & & 9205 & 29 \\
\hline & & clutiman & & 10007 & 146 \\
\hline & & director & & 6 & 1.58 \\
\hline & & minister & & 37 & 17 \\
\hline & & premier & & 7 & 8 \\
\hline \multirow[t]{7}{*}{ minn } & lory & story & & ) & 3 \\
\hline & & club & & 6 & 4 \\
\hline & & age: & & 18 & 3 \\
\hline & & molher & & 4 & 5 \\
\hline & & & bial & 4 & 4 \\
\hline & & & youmi & 258 & 12 \\
\hline & & & older & 18 & 4 \\
\hline
\end{tabular}

Migure 2. Example pairs of felated tems.

slette, 1992): 5

3 In another series of experiments (Strzalkowski \& Vauthey, 1992) we used a Mutual Infomation based chassification formula (e.g. Church and Hanks, 1990; Hlindle, 1990), but we found it less effective for diverse databases, such as WSJ. 
$\operatorname{SIM}\left(x_{1}, x_{2}\right)=\frac{\sum_{a t t} M I N(W([x, a t t]), W([y, a t t])}{\sum_{a t t} M A X(W([x, a t t]), W([y, a t t])}$

with

$W([x, y])=\operatorname{CEW}(x)^{*} \log \left(f_{x, y}\right)$

$\operatorname{GEW}(x)=1+\sum_{y}\left(\frac{\frac{f_{x, y}}{n_{y}} * \log \left(\frac{f_{x, y}}{n_{y}}\right)}{\log (N)}\right)$

In the above, $f_{x, y}$ stands for absolute frecpuency of pair $[x, y]$ in the corpus, $n_{y}$ is the frequency of term $y$, and $N$ is the number of single-word terms.

In order to generate better similarities, we require that words $x_{1}$ and $x_{2}$ appear in at least $M$ distinct common contexts, where a common context is a couple of pairs $\left[x_{1}, y\right]$ and $\left[x_{2}, y\right]$, or $\left[y, x_{1}\right]$ and $\left[y_{,} x_{2}\right]$ such that they each occurred at least $K$ times. Thus, banana and Baltic will not be considered for similarity relation on the basis of their occurrences in the common context of republic, no matter how frequent, unless there are $M-1$ other such common contexts comparably frequent (there wasn't any in TREC's WSJ database). For smaller or narrow domain databases $M=2$ is usually sufficient, e.g., CACM database of computer science abstracts. For large databases covering at diverse subject matter, like WSJ or SJMN (San Jose Mercury News), we used $M \geq 5 .{ }^{6}$ This, however, turned out not to be sulficient. We would still generate fairly strong similarity links between terms such as aerospace and pharmaceutical where 6 and more common contexts were found, even after a number of common contexts, such as company or market, have already been rejected because they were paired with too many different words, and thus had a dispersion ratio too high. The remaining common contexts are listed in Figure 3, along with their GEW scores, all occurring at the head (left) position of at pair.

\begin{tabular}{|lccc|}
\hline CONIEXT & GEW & \multicolumn{2}{c}{ frequency with } \\
fim & & aerospace & pharmacentical \\
industry & 0.58 & 9 & 22 \\
sector & 0.61 & 84 & 56 \\
concen & 0.50 & 130 & 9 \\
analyst & 0.62 & 23 & 115 \\
division & 0.53 & 36 & 88 \\
giant & 0.62 & 15 & 12 \\
\hline
\end{tabular}

Figure 3. Common (head) contexts for aerospace and pharmaceutical.

- For example tanama and Dominican were found to have two common contexts: republic and plant, although this second occurred in apparently different senses in Dominican plant and banana plant.
When analyzing Figure 3, we should note that while some of the GEW weights are guite low (GEW takes values between 0 and 1 ), thus indicating a low importance context, the frequencies with which these contexts occurred with both terms were high and balanced on both sides (c.g., concern), thus adding to the strength of association. To filter out such cases we established thresholds for admissible values of GEW factor, and disregarded contexts with entropy weights falling below the threshold. In the most recent experiments will WSJ texts, we found that 0.6 is a good threshold. We also observed that clustering head terms using their modifiers as contexts converges faster and gives generally more reliable links than when mod terms are clustered using heads as context (c.g., in the above example). In our experiment with the WSJ database, we found that an occurrence of a common head context needs to be considered as contributing less to the total context count than an occurrence of a common mod context: we used 0.6 and 1 , respectively. Using this formula, terms man and boy in Figure 2 share 5.4 contexts ( 4 head contexts and 3 mod contexts).

Initially, term similarities are organized into clusters around it centroid term. Figure 4 shows top 10 elements (sorted by similarity value) of the cluster for president. Note that in this case the SIM value drops suddenly after the second element of the cluster. Changes in SIM value are used to determine cut-off points for clusters. The role of GTS factor will be explained later. Sample clusters obtained from approx. 250 MByte (42 million words) subset of WSJ (years 1990-1992) are given in Table 1.

It may be worth pointing out that the similarities are calculated using term co-oceurrences in syntactic rather than in document-size contexts, the latter being the usual practice in non-linguistic clustering (e.g., Sparck Jones and Barber, 1971; Crouch, 1988; Lewis and Crolt, 199()). Although the two methods of term clustering may be considered mutually complementary in certain situations, we believe that more and stronger associations can be obtained through syntactic-context clustering, given sufticient amount of data and a reasonably accurate syn-

\begin{tabular}{|c|c|c|c|}
\hline \multirow[t]{10}{*}{$\begin{array}{l}\text { Clin'TROIl) } \\
\text { presiclent }\end{array}$} & TliRM & SIM & $\begin{array}{l}0.15 \\
0.0011\end{array}$ \\
\hline & director & 0.2481 & 0,0017 \\
\hline & dhaimman & 0.2449 & 0.0028 \\
\hline & office & 0.1689 & 0.0010 \\
\hline & manage & 0.1656 & 0.0007 \\
\hline & executive & 0.1626 & 0.0012 \\
\hline & official & 0.1612 & 0.0008 \\
\hline & head & 0.1564 & 0.0018 \\
\hline & member & 0.1506 & 0.0014 \\
\hline & lead & 0.1311 & 0.00009 \\
\hline
\end{tabular}

IHigure 4. A cluster for president. 


\begin{tabular}{|c|c|}
\hline word & cluster \\
\hline takeover & merge, buy-out, acquirc, bid \\
\hline benefit & compensate, aid, expense \\
\hline capital & cassh, fishd, money \\
\hline staff & personnel, employee, force \\
\hline attract & lure, draw, woo \\
\hline sensitive & crucial, difficull, critical \\
\hline speculate & rumor, uncertainfy, tension \\
\hline president & director, chairman \\
\hline vice & deputy \\
\hline oullook & forecast, prospect, trond \\
\hline$l a w$ & rule, policy, legislate, bill \\
\hline earnings & profit, reveruse, income \\
\hline portfolio & asset, inve'st, loan \\
\hline inflate & growth, demand, earnings \\
\hline industry & business, company, marke! \\
\hline growth & increase, rise, gain \\
\hline firm & bank, concern, group, unit \\
\hline environ & climate, condition, sibuation \\
\hline debt & loan, secure, bond \\
\hline lawyer & attorney \\
\hline counsel & attorney, administrator, secrotary \\
\hline compute & machine, soffucare, erfuipment \\
\hline competitor & rival, competition, buyer \\
\hline alliance & partmership, venture, consoritum \\
\hline$b i g$ & large, mojor, huge, significant \\
\hline fight & battle, attack, war, challenge \\
\hline base & facile, source, reserve, stupport \\
\hline shareholder & $\begin{array}{l}\text { creditor, customer, client } \\
\text { investor, stockholder }\end{array}$ \\
\hline
\end{tabular}

Table 1. Selected clusters obtained from syntatic contexts, derived from approx. 40 million words of WSJ text, with weighted Jaceand formuln.

tactic parser. ${ }^{7}$

${ }^{7}$ Non-syntactic contexts cross sentence boundaries with no fuss, which is helpful with shor, succinct doctments (such as $\mathrm{C} \wedge \mathrm{M}$ ) abstracts), but less so with longer texts; see also (Grishman et al., 1086)

\section{QUERY EXI'ANSION}

Similarity relations are used to expand user queries with new terms, in an attempt to make the final search query more compreluensive (adding synonyms) and/or more pointed (adding specializations). It follows that not all similarity relations will be equally useful in query expansion, for instance, complementary and antonymous relations like the one between Australian and Canadiam, accept and reject, or even generalizations like from aerospace to industry may actually hatrm system's performance, since we may end up retrieving many irclevant documents. On the other hand, database search is likely to miss relevant decuments if we overlook the latet that vice director can also be depury director, or that takeover can atso be merge, buy-out, or acquisition. We noted that an average sel of similarities gencrated from a lext corpus contains about as many "good" relations (synonymy, specialization) as "bad" relations (antonymy, complementattion, generalization), as seen from the query expansion viewpoint. Therefore any attempl to separate these two classes and to increase the proportion of "good" relations should result in improved retrieval. This has indeed been confirmed in our experiments where a relatively crude filter has visibly increased retricval precision.

In order to create an appropriate filter, we devised a global term specificity measure (GTS) which is calculated for each term across all contexts in which it occurs. The general philosophy here is that a more specific word/phrase would have a more limited use, i.e., a more specific term would appear in fewer distinet contexts. In this respect, GTS is similar to the standard inverted documen frequency $(i d f)$ measure except that term frequency is measured over syntactic units mather than document size units. Terms with higher GTS values atre generally considered more specific, but the specificity comparison is only meaningful for terms which are already known to be similar. We believe that measuring term specificity over document-size contexts (e.g., Sparck Jones, 1972) may not be appropriate in this case. In particular, syntax-based contexts allow for processing texts without any internal clocument structure.

The new function is calculated according to the following formula:

$\operatorname{CTS}(w)= \begin{cases}I C_{L}(w) * I C_{R}(w) & \text { if both exist } \\ I C_{R}(w) & \text { if only } I C_{R}(w) \text { cxists } \\ I C_{L}(w) & \text { otherwise }\end{cases}$

where (with $n_{w}, d_{w}>0$ ):

$I C_{L}(w)=I C(\mid w, 1)=\frac{n_{w}}{a_{w}\left(n_{w}+d_{w}-1\right)}$
$I C_{R}(w)=I C(1, w \mid)=\frac{n_{w}}{a_{w}\left(n_{w}+d_{w}-1\right)}$

In the ahove, $d_{w}$ is dispersion of term $w$ understood ats the number of distinct contexts in which $w$ is found. For any two terms $w_{1}$ and $w_{2}$, and a constant $\delta_{1}>1$, if $\operatorname{CTS}\left(w_{2}\right) \geq \delta_{1} * \operatorname{GTS}\left(w_{1}\right)$ then $w_{2}$ is considered more specific than $w_{1}$. In addition, if $S i M_{n o m}\left(w_{1}, w_{2}\right)=\sigma>0_{1}$, where $0_{1}$ is an empirically 
established threshold, then $w_{2}$ can be added to the query containing term $w_{1}$ with weight $\sigma^{*} \omega,{ }^{8}$ where $\omega$ is the weight $w_{2}$ would have if it were present in the query. Similarly, if $\operatorname{GTS}\left(w_{2}\right) \leq \delta_{2} * \operatorname{GTS}\left(w_{1}\right)$ and $S I M_{\text {narm }}\left(w_{1}, w_{2}\right)=\sigma>\theta_{2}$ (with $\delta_{2}<\delta_{1}$ and $\theta_{1}<\theta_{2}$ ) then we may consider $w_{2}$ as synonymous to $w_{1}$. All other relations are discarded. For example, the following were obtained from the WSJ training database:

$$
\begin{array}{ll}
\text { GTS (takeover) } & =0.00145576 \\
\text { GTS (merge) } & =0.00094518 \\
\text { GTS }(\text { buy-out) } & =0.00272580 \\
\text { GTS (acquire) } & =0.00057906
\end{array}
$$

with

$$
\begin{array}{ll}
\text { SIM (takeover, merge) } & =0.190444 \\
\text { SIM (takeover,buy-out) } & =0.157410 \\
\text { SIM (takeover, acquire) } & =0.139497 \\
\text { SIM (merge, buy-out) } & =0.133800 \\
\text { SIM (merge, acquire) } & =0.263772 \\
\text { SIM (buy-out, acquire) } & =0.109106
\end{array}
$$

Therefore both takeover and buy-out can be used to specialize merge or acquire. With this filter, the relationships between takeover and buy-out and between merge and acquire are either both discarded or accepted as synonymous. At this time we are unable to tell synonymous or near synonymous relationships from those which are primarily complementary, e.g., man and woman.

Filtered similarity relations create a domain map of terms. At present it may contain only two types of links: equivalence (synonymy and near-synonymy) and subsumption (specification). Figure 5 shows a small fragment of such map derived from lexical relation computed from WSJ database. The domain map is used to expand user queries with related terms, either automatically or in a feedback mode by showing the user appropriate parts of the map.
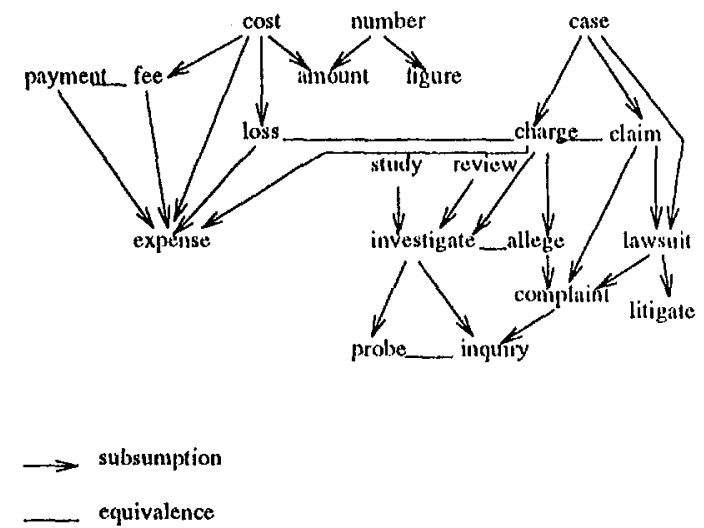

Figure 5. A fragment of the domain map network. Note the emerging senses of 'charge' as 'expense' and 'allege'.

For TREC-2 we used $\theta=0.2 ; 8$ varied between 10 and 100 .
We should add that the query expansion (in the sense considered here, though not quite in the same way) has been used in information retrieval research before (e.g., Sparck Jones and Tait, 1984; Harman, 1988), usually with mixed results. The main difference between the current approach and those previous attempts is that we use lexico-semantic evidence for selecting extra terms, while they relied on term co-occurrence within the same documents. In fact we consider these to methods complementary with the latter being more appropriate for automatic relevance feedback. An alternative query expansion to is to use term clusters to create new terms, "metaterms", and use them to index the database instert (e.g., Crouch, 1988; Lewis and Croft, 1990). We found that the query expansion approach gives the system more flexibility, for instance, by making room for hypertextstyle topic exploration via user feedback.

\section{CONCLUSIONS}

We discussed selected aspects our information retrieval system consisting of an advanced NLP module and a 'standard' statistical core engine. In this paper wo concentrated on the problem of automatic generation of lexical correlations among terms which (along with appropriate weighting scheme) represent the content of both the database documents and the user queries. Since a successful retrieval relies on actual term matches between the queries and the documents, it is essential that any lexical alternatives of describing a given topic are taken into account. In our system this is achieved through the expansion of user's queries with related terms: we add equivalent and more specific terins, Lexical relations between terms are calculated directly from the database and stored in the form of a domain map, which thus acts as a domain-specilic thesaurus. Query expansion can be done in the user-feedback mode (with user's assistince) or atomatically. In this latter case, local context is explored to assure meaningful expansions, i.e., to prevent e.g., expanding 'charge' with 'expense' when 'allege' or 'blame' is meant, as in the following example query:

Documents will report on corruption, incompetence, or inefficiency in the management of the United Nation's stall, Allegations of management failings, as well as retorts to such charges are belevant.

Many problems remain, however, we attempled to demonstrate that the archiecture deseribed liere is nonetheless viable and has practical significance. More advanced NLP techniques (including semantic analysis) may prove to be still more eflective, in the future, however their enormous cost limits any experimental evidence to small scale tests (e.g., Mauldin, 1991).

\section{ACKNOWLEDGEMENTS}

We would like to thank Donna Harman of NIST for making her PRISE system available to us. We would also like to thank Ralph Weischedel and Heidi Fox of BBN for providing and assisting in the use of the part of speech tagger. This paper is based upon work supported by the Advanced Research Project Agency under Contract 
N00014-90-J-1851 from the Office of Naval Research, under Contract N00600-88-D-3717 from PRC Inc., and the National Science Foundation under Grant IRI-9302615. We also acknowledge support from the Canadian Institute for Robotics and Intelligent Systems (IRIS).

\section{REIERENCES}

Church, Kenneth Ward and Lanks, Parrick. 1990. "Word association norms, mutual information, and lexicography." Computational Linguistics, 16(1), MIT Press, pp. 22-29.

Crouch, Carolyn J. 1988. "A cluster-based approach 10 thesturus construction." Proceedings of ACM SIGIR-88, pl. 309-320.

Grefenstete, Gregory. 1992. "Use of Syntactic Context To Produce Term Association Lists for Text Retrieval." Proceedings of SIGIR-92, Copenhagen, Denmark. pp. 89-97.

Grishman, Ralph, Lynette Hirschman, and Ngo T. Nhan. 1986. "Discovery procedures for sublanguage selectional patterns: initial experiments". Compatational Linguistics, 12(3), pp. 205-215.

Harman, Donna. 1988. "Towards interactive query expansion." Proceedings of ACM SIGIR-88, $\mathrm{mp}$. 321-331.

Hindle, Donald. 1990. "Noun classification from predicate-argument structures." Proc. 28 Meeting of the ACL, Pittshurgh, PA, pp. 268-275.

Lewis, David D. and W. Bruce Croft. 1990. "Term Clustering of Syntactic Phrases". Proceedings of ACM SIGIR-90, pp. 385-405.

Mauldin, Michael. 1991. "Retrieval Performance in Ferret: A Conceptual Information Retrieval System." Proceedings of ACM SIGIR-91, pp, 347-355.

Salton, Gerard. 1989. Antomatic Text Processing: the transformation, analysis, and retrieval of information by computer. Addison-Wesley, Reading, MA.

Sparck Jones, Karen. 1972. "Statistical interpretation of term specificity and its application in retrieval." Journal of Docamentation, $28(1)$, pp. $11-20$.

Sparck Jones, K. and E. O. Barber. 1971. "What makes automatic keyword classification effective?" Journal of the American Socicty for Information Science. May-June, pp. 166-175.

Sparck Jones, K. and J. I. Tait, 1984. "Automatic search term vatiant generation," Journal of Docmmentation. $40(1)$, p). 50-66.

Stræalkowski, Tomek and Barbara Vauthey. 1992. "Information Retrieval Using Robust Naural Language Processing." Proc. of the 30th ACL Mecting, Newark, DE, June-July. pp. 104-111.

Stralkowski, Tomek. 1992. "TTP: A Fast and Robust Parser for Natural Language." Proceedings of the 14th International Conference on Computational Linguistics (COLING), Nantes, Franee, July 1992. pr. 198-204.

Strzalkowski, Tomek. 1993. "Robust Text Processing in Automated Information Retrieval." Proc. of $\mathrm{ACL}$. sponsored workshop on Very Large Corpora. Ohio State Univ. Columbus, Junc 22.

Straatkowski, Tomek. 1994. "Drument Representation in Natural Language Text Retrieval." To appear in proced dings of ARPA Human Language Technology Workshop, Princeton, NJ. March 8-11.

Stratkowski, Tomek and Jose Perez-Carballo. 1994. "Recent Developments in Natural Language Text Retrieval." To appea in proceedings of Second Tex1 Retrieval Conference (TREC-2), (iathershurg, Md, Allgust 30 - September 1, 1993.

Stralkowski, Tomek, and Peter Scheyen. 1993. "Evaluittion of TTP Parser: a preliminary report." Proceedings of International Workshop on Parsing Teclmologies (IWP'[-93), Tilburg, Netherlands and Durbuy, Belgium, August 10-13.

\section{APl'LNDIX: An example query}

The following is an example information request (based on TREC's topic 113) and the resulting query. Except for its inverted document frequency score, each term has a "confidence level" weight which is set to 1.0 it the term is found in the teser's query, and is less than 1.0 if the term is added through an expansion from the clomain map. Only non-negated terms with idf of $6.0 \mathrm{or}$ greater are included.

citle> New Space Satellite Applications

<descr Document will report on non-traditional apsplications of space satellife technology.

<nars A relevant document will diseuss more recent or emerging applications of space satellite technology. NO'T relevant are such "tralitional" or early sittellite age usages as INTELSAT tansmission of voice and data communications for telephone companies or program feeds for established television networks. Also NO'J relevant are such established uses of satellites as militay communicatous, earth mineral resource mapping, and support of weather forecasling. A fow examples of newer applications are the buileling of private satellite networks for transfer of business data, fucsimile transmission of newspapers to be jrinted in multiple locations, and direc broadcasting of TV signals. The underlying purpose of this topic is to collect information on recent or emerging tremels in the application of space satellite techology.

\begin{tabular}{|c|c|c|}
\hline$T E K M$ & HOF & Whitill] \\
\hline applytecunip & 18.402237 & 0.458606 \\
\hline satellite+latest & 18.402237 & $0.25405 \mathrm{x}$ \\
\hline televisiontsignal & 18.402237 & 0.359777 \\
\hline television-rlifect & 18.402237 & 0.350777 \\
\hline apply+ecpuip & 18.402237 & 0.458666 \\
\hline broakdeastitidirect & 16.402237 & 1.000000 \\
\hline location+multiple & 16.402237 & 1.000000 \\
\hline broatdeast+signal & 16.080309 & 1.000000 \\
\hline suppont+forecast & 15.817275 & 1.0000000 \\
\hline datatbusiness & 15.817275 & 1.000000 \\
\hline Forecast-internal & 15.402238 & 0.28 .3029 \\
\hline transfertinfom & 15.232312 & 0.5119 \\
\hline transfortdata & 14.817275 & 1.0000 \\
\hline figure+business & 1.1 .594883 & 0.4536 \\
\hline
\end{tabular}




$\begin{array}{lll}\text { teclnology+satellite } & 14.495347 & 1.000000 \\ \text { transmit+facsimile } & 14.402238 & 1.000000 \\ \text { equip+satellite } & 14.232312 & 0.458666 \\ \text { signal+broadcast } & 13.701797 & 0.441993 \\ \text { signal+tv } & 13.701797 & 1.000000 \\ \text { signal+television } & 13.594883 & 0.813987 \\ \text { news+business } & 13.495347 & 0.352291 \\ \text { network+satellite } & 13.154310 & 1.000000 \\ \text { develop+network } & 12.942806 & 0.409144 \\ \text { non+traditional } & 12.758382 & 1.000000 \\ \text { inform+business } & 12.729813 & 0.511940 \\ \text { apply+technology } & 12.471500 & 1.000000 \\ \text { build+network } & 11.212413 & 1.000000 \\ \text { facsinile } & 10.217362 & 1.000000 \\ \text { usage } & 9.902391 & 1.000000 \\ \text { newer } & 9.306841 & 1.000000 \\ \text { elderly } & 8.202565 & 0.361246 \\ \text { feed } & 7.802325 & 1.000000 \\ \text { satellite } & 7.567767 & 1.000000 \\ \text { underly } & 7.370192 & 1.000000 \\ \text { transmit } & 7.299606 & 1.000000 \\ \text { multiple } & 7.241736 & 1.000000 \\ \text { broatcast } & 7.019614 & 1.000000 \\ \text { location } & 6.992316 & 1.000000 \\ \text { print } & 6.351709 & 1.000000 \\ \text { space } & 6.226376 & 1.000000 \\ \text { transfer } & 6.155497 & 1.000000 \\ \text { collect } & 6.126113 & 1.000000 \\ \text { signal } & 6.080873 & 1.000000 \\ \text { phone } & 6.072441 & 0.6634414 \\ \text { tv } & 6.003761 & 1.000000\end{array}$

